\title{
SQUARE INTEGRABLE HARMONIC FUNCTIONS ON PLANE REGIONS
}

\author{
MITSURU NAKAI and LEO SARIO
}

Let $R$ be a thin horizontal elastic plate clamped along its border. We denote by $\beta_{R}(z, \zeta)$ the deflection of $R$ at $z \in R$ under a point load at $\zeta \in R$, that is, the biharmonic Green's function on $R$ with the pole at $\zeta$. The function is characterized by $\Delta_{z}^{2} \beta_{R}(z, \zeta)=2 \pi \delta_{\zeta}$ on $R$, with $\delta_{\zeta}$ the Dirac measure at $\zeta$, and by the conditions $\beta_{R}(z, \zeta)=\partial \beta_{R}(z, \zeta) / \partial n_{z}=0$ at the boundary $\partial R$ of $R$ (e.g., Bergman-Schiffer [1]). Accordingly, it is customary to assume that the boundary $\partial R$ of $R$ relative to the complex plane $\boldsymbol{C}$ is smooth. If $\boldsymbol{R}$ is an arbitrary plane region, a natural procedure is to define $\beta_{R}(z, \zeta)$ for $z \in R$ as the directed limit

$$
\lim _{\Omega \rightarrow R} \beta_{\Omega}(z, \zeta)
$$

where $\{\Omega\}$ is the directed set of regular subregions, i.e., relatively compact subregions $\Omega$ of $R$ with smooth boundaries $\partial \Omega$. We denote by $O_{\beta}$ the family of plane regions $R$ for which (1) is divergent for some $\zeta \in R$. The purpose of the present paper is to give a complete characterization of $O_{\beta}$ as follows:

$1^{\circ}$. A plane region $R \in O_{\beta}$ if and only if the complement $C-R$ of $R$ does not contain any noncollinear triple of points (and hence e.g. $C-\{0,1, i\} \notin O_{\beta}$ !).

$2^{\circ}$. If a plane region $R \notin O_{\beta}$, then $\beta_{R}(z, \zeta)=\lim _{\Omega \rightarrow R} \beta_{\Omega}(z, \zeta)$ is symmetric and continuous on $R \times R$, the convergence is uniform on every compact subset of $R \times R$, and $z \rightarrow \beta_{R}(z, \zeta)$ is biharmonic on $R-\zeta$.

$3^{\circ}$. There exist plane regions $R$ which are "unstable" in the sense that (1) is divergent for some $(z, \zeta) \in R \times R$ but convergent for some other $(z, \zeta) \in R \times R$. Such unstable regions $R$ are characterized by the existence of a line $l(R)$ such that $C-R$ is a proper subset of $l(R)$ consisting of at least two points, and (1) is divergent at e.g., $(\zeta, \zeta)$ for any $\zeta \notin l(R)$ and convergent at every $(z, \zeta) \in(l(R) \times l(R)) \cap(R \times R)$.

We denote by $H_{2}(R)$ the closed subspace of $L_{2}(R)$ consisting of square integrable harmonic functions on $R$. To prove $1^{\circ}-3^{\circ}$, we shall make essential use of the follow-

MOS Classification 31B30.

The work was supported by the National Science Foundation, Grant MCS 77-16198, University of California, Los Angeles. 
ing results obtained in [3]. Let $H(\Omega)$ be the class of harmonic functions on $\Omega$ and denote by $(,)_{\Omega}$ the inner product in $L_{2}(\Omega)$. The function $H_{\Omega}(z, \zeta)=\Delta_{z} \beta_{\Omega}(z, \zeta)$ is referred to as the $\beta$-density on $\Omega$, characterized by $H_{\Omega}(z, \zeta)+\log |z-\zeta| \in H(\Omega)$ as a function of $z$, and by $\left(H_{\Omega}(\cdot, \zeta), u\right)_{\Omega}=0$ for every $u \in H_{2}(\Omega)$. Then $\beta_{\Omega}(z, \zeta)=$ $\left(H_{\Omega}(\cdot, z), H_{\Omega}(\cdot, \zeta)\right)_{\Omega}$ and

$$
\left|\beta_{\Omega^{\prime}}(z, \zeta)-\beta_{\Omega}(z, \zeta)\right| \leqq\left\|H_{\Omega^{\prime}}(\cdot, z)-H_{\Omega}(\cdot, z)\right\|_{R} \cdot\left\|H_{\Omega^{\prime}}(\cdot, \zeta)-H_{\Omega}(\cdot, \zeta)\right\|_{R}
$$

on $\Omega \times \Omega$ for every regular subregion $\Omega^{\prime}$ with $\Omega \subset \Omega^{\prime} \subset R$; here $\|\cdot\|_{R}$ is the norm in $L_{2}(R)$, and we have set $H_{\Omega}(z, \zeta)=0$ for $(z, \zeta) \notin \Omega \times \Omega$. In particular, we have

$$
\beta_{\Omega^{\prime}}(\zeta, \zeta)-\beta_{\Omega}(\zeta, \zeta)=\left\|H_{\Omega^{\prime}}(\cdot, \zeta)-H_{\Omega}(\cdot, \zeta)\right\|_{R}^{2}=\left\|H_{\Omega}(\cdot, \zeta)\right\|_{R}^{2}-\left\|H_{\Omega}(\cdot, \zeta)\right\|_{R}^{2} .
$$

Thus the limit (1) exists if and only if

$$
\lim _{\Omega \rightarrow R}\left\|H_{\Omega}(\cdot, \zeta)\right\|^{2}<+\infty .
$$

This in turn is equivalent to the existence of an $H_{R}(\cdot, \zeta)$ on $R$ such that $H_{R}(z, \zeta)+$ $\log |z-\zeta| \in H(R)$ as a function of $z$ and $\left(H_{R}(\cdot, \zeta), u\right)=0$ for every $u \in H_{2}(R)$; in this case, $\lim _{\Omega \rightarrow R}\left\|H_{R}(\cdot, \zeta)-H_{\Omega^{\prime}}(\cdot, \zeta)\right\|_{R}=0$ and $\beta_{R}(z, \zeta)=\left(H_{R}(\cdot, z), H_{R}(\cdot, \zeta)\right)_{R}$.

We will see that the orthogonal complement $H_{2}(R)_{\zeta}^{\perp}$ of $H_{2}(R)$ in $H_{2}(R-\zeta)$ is either $\{0\}$ or $\boldsymbol{R} H_{R}(\cdot, \zeta)$, where $\boldsymbol{R}$ is the field of real numbers. Accordingly, the essential point is to determine the pairs $(R, \zeta)$ of plane regions $R$ and their points $\zeta \in R$ such that $\operatorname{dim} H_{2}(R-\zeta)=\operatorname{dim} H_{2}(R)+1$. Thus we are led to study the Hilbert space $H_{2}(R)$. It is locally bounded and therefore has a reproducing kernel $h_{R}(z, \zeta)$ characterized by $u(\zeta)=\left(u, h_{R}(\cdot, \zeta)\right)_{R}$ for every $u \in H_{2}(R)$. It is seen that $h_{R}(\cdot, \zeta)=$ $\Delta_{\zeta} H_{R}(\cdot, \zeta)$ if $H_{R}(\cdot, \zeta)$ exists (cf. e.g. Garabedian [2]); we will, however, not make use of this fact in the present work.

In nos. 1-5 we study the dimension of $H_{2}(R)$ and give a complete characterization of those plane regions $R$ for which $\operatorname{dim} H_{2}(R)=0$. We then proceed to $H_{2}(R-\zeta)$ and, in nos. 6-7, characterize those plane regions $R$ for which $\operatorname{dim} H_{2}(R-\zeta)=$ $\operatorname{dim} H_{2}(R)+1$ for every $\zeta \in R$, for some $\zeta \in R$, or for $n o \zeta \in R$. For the first case we study, in nos. 8-11, the continuity of $H_{R}(\cdot, \zeta)$ and the uniformity of the convergence $H_{\Omega}(\cdot, \zeta) \rightarrow H_{R}(\cdot, \zeta)$ with respect to $\zeta$. That assertions $1^{\circ}-3^{\circ}$ follow from these considerations will be briefly discussed in the final no. $\mathbf{1 2}$.

We close this introduction by stressing once more that the class $O_{\beta}$ is not conformally invariant and not even invariant under Möbius transformations. In fact, the regions $\boldsymbol{C}-\{0,1, i\} \notin O_{\beta}$ and $\boldsymbol{C}-\{0,1,2\} \in O_{\beta}$ are equivalent by the Möbius transformation $(z, 0,1, i)=(w, 0,1,2)$.

1. Suppose $u(z)$ is harmonic in a punctured disk $\Delta_{0}(\zeta, \varrho): 0<|z-\zeta|<\varrho$ about a point $\zeta \in \boldsymbol{C}$ (the finite complex plane). Then $u(z)$ has the Laurent expansion

$$
u(z)=\operatorname{Re}\left(-c \log (z-\zeta)+\sum_{n=-\infty}^{\infty} a_{n}(z-\zeta)^{n}\right)
$$


in $\Delta_{0}(\zeta, \varrho)$, with $c \in \boldsymbol{R}$ (the field of real numbers) and $a_{n} \in \boldsymbol{C}$. It is readily seen that $u$ is square integrable in $\Delta_{0}(\zeta, \bar{\varrho})(\bar{\varrho} \in(0, \varrho))$ if and only if $a_{n}=0$ for every negative $n$ :

$$
u(z)=\operatorname{Re}\left(-c \log (z-\zeta)+\sum_{n=0}^{\infty} a_{n}(z-\zeta)^{n}\right) .
$$

Next suppose $u(z)$ is harmonic in a punctured disk $\Delta_{0}(\infty, \varrho): \varrho<|z|<+\infty$ about the point $\infty$ at infinity. Then the Laurent expansion of $u(z)$ is given by

$$
u(z)=\operatorname{Re}\left(c \log z+\sum_{n=-\infty}^{\infty} a_{n} z^{-n}\right)
$$

in $\Delta_{0}(\infty, \bar{\varrho})(\varrho<\bar{\varrho})$ where $\mathbf{c} \in \boldsymbol{R}$ and $a_{n} \in \boldsymbol{C}$. Again it is clear that $u$ is square integrable if and only if $c=a_{n}=0$ for every integer $n \leqq 1$ :

$$
u(z)=\operatorname{Re}\left(\sum_{n=2}^{\infty} a_{n} z^{-n}\right),
$$

and in this case $u$ is also harmonic at $\infty$ with $u(\infty)=0$.

For convenience we denote by $l_{\zeta}(z)$ the normalized logarithmic pole $-\log |z-\zeta|$ at $\zeta \in \boldsymbol{C}$. The Laurent expansion of $l_{\zeta}(z)$ is

$$
l_{\zeta}(z)=\operatorname{Re}\left(-\log z+\sum_{n=1}^{\infty} \frac{\zeta^{n}}{n} z^{-n}\right)
$$

in $\Delta_{0}(\infty,|\delta|)$. Note that the coefficient of $z^{-1}$ is $\zeta$.

2. Let $\zeta$ be the set of $m$ distinct points $\zeta_{j}$ in $C(j=1, \ldots, m)$ and consider the region $R_{\zeta}=C-\zeta$. The matrix

$$
A=A(\zeta)=\left(\begin{array}{cccc}
1 & 1 & \ldots & 1 \\
\operatorname{Re} \zeta_{1} & \operatorname{Re} \zeta_{2} & \ldots & \operatorname{Re} \zeta_{m} \\
\operatorname{Im} \zeta_{1} & \operatorname{Im} \zeta_{2} & \ldots & \operatorname{Im} \zeta_{m}
\end{array}\right)
$$

associated with the region $R_{\zeta}$ will be instrumental in our reasoning. We shall also use the column vector $\boldsymbol{t}$ whose components are $t_{1}, t_{2}, \ldots, t_{m}$ in $\boldsymbol{R}$. Let $S=S(\zeta)$ be the vector space of solution vectors $\boldsymbol{t}$ of the equation $A \boldsymbol{t}=\mathbf{0}$ where $\mathbf{0}$ is the transpose of $(0,0,0)$. Then

$$
\operatorname{dim} S(\zeta)=m-\operatorname{rank} A(\zeta)
$$

With each column vector $\boldsymbol{t}$ we associate $h_{t}=\sum_{j=1}^{m} t_{j} l_{\zeta_{j}}$. We will show that $\boldsymbol{t} \rightarrow h_{\boldsymbol{t}}$ is a linear bijection: $S \rightarrow H_{2}\left(R_{\zeta}\right)$, so that

$$
\operatorname{dim} H_{2}\left(R_{\zeta}\right)=m-\operatorname{rank} A(\zeta) .
$$

First we prove that $h_{t} \in H_{2}\left(R_{\zeta}\right)$ if $t \in S$. It is clear that $h_{t}$ belongs to $H\left(R_{\zeta}\right)$ and is square integrable over some $\Delta_{0}\left(\zeta_{j}, \varrho_{j}\right)$ for every $j=1, \ldots, m$. By (7) we see that

$$
h_{t}(z)=\operatorname{Re}\left(-\left(\sum_{j=1}^{m} t_{j}\right) \log z+\left(\sum_{j=1}^{m} \zeta_{j} t_{j}\right) z^{-1}+\sum_{n=2}^{\infty} \alpha_{m} z^{-n}\right)
$$


where $\alpha_{n}=n^{-1} \sum_{j=1}^{m} \zeta_{j}^{n} t_{j}$. Since $A \boldsymbol{t}=\mathbf{0}$, the coefficients of $\log z$ and $z^{-1}$ of $h_{t}(z)$ must vanish and we obtain $h_{t}(z)=\operatorname{Re}\left(\sum_{n=2}^{\infty} \alpha_{n} z^{-n}\right)$, which by (6) shows that $h_{t}$ is also square integrable over some $\Delta_{0}(\infty, \varrho)$. Since $C-\Delta_{0}(\infty, \varrho) \cup \sum_{j=1}^{m} \Delta_{0}\left(\zeta_{j}, \varrho_{j}\right)$ is compact we finally conclude that $h_{t} \in H_{2}\left(R_{\zeta}\right)$, that is, $t \rightarrow h_{t}$ is a well defined mapping: $S \rightarrow H_{2}\left(R_{\zeta}\right)$. Since $\left\{l_{\zeta_{j}}\right\}_{j=1, \ldots, m}$ is a linearly independent family, we see that $\boldsymbol{t} \rightarrow h_{t}$ is a linear injection of $S$ into $H_{2}\left(R_{\zeta}\right)$.

Next we prove that it is surjective. Choose an arbitrary $u \in H_{2}\left(R_{\zeta}\right)$. By (5) we have

$$
u(z)=\operatorname{Re}\left(-t_{j} \log \left(z-\zeta_{j}\right)+\sum_{n=0}^{\infty} a_{j n}\left(z-\zeta_{j}\right)^{n}\right)
$$

in a certain $\Delta_{0}\left(\zeta_{j}, \varrho_{j}\right)(j=1, \ldots, m)$. This determines the column vector $t$ whose components are $t_{1}, \ldots, t_{m}$. Observe that $l_{\zeta_{j}}-l_{\zeta_{m}} \in H\left(\hat{\boldsymbol{C}}-\left\{\zeta_{j}, \zeta_{m}\right\}\right)(j=1, \ldots, m)$ and vanishes at $\infty$; here $\hat{\boldsymbol{C}}=\boldsymbol{C} \cup\{\infty\}$, the extended complex plane. Consider the function

$$
h(z)=u(z)-\sum_{j=1}^{m-1} t_{j}\left(l_{\zeta_{j}}(z)-l_{\zeta_{m}}(z)\right) .
$$

By (6) and the above remark, we see that $h \in H\left(\hat{\boldsymbol{C}}-\zeta_{m}\right)$, and

$$
h(z)=\operatorname{Re}\left(\left(\sum_{j=1}^{m} t_{j}\right) l_{\zeta_{m}}(z)+\sum_{n=0}^{\infty} a_{m n}\left(z-\zeta_{m}\right)^{n}\right)
$$

in a certain $\Delta_{0}\left(\zeta_{m}, \varrho_{m}\right)$. We denote by $C$ the boundary of the disk $\Delta\left(\zeta_{m}, \varrho_{m} / 2\right):\left|z-\zeta_{m}\right|<$ $\varrho_{m} / 2$. Then $h$ is harmonic on $\hat{\boldsymbol{C}}-\bar{\Delta}\left(\zeta_{m}, \varrho_{m} / 2\right)$ and the Gauss theorem assures the vanishing of the flux of $h$ across $C$. On the other hand this flux is the sum of

$$
\left(\sum_{j=1}^{m} t_{j}\right) \int_{C} * d l_{\zeta_{m}}(z)=2 \pi \sum_{j=1}^{m} t_{j}
$$

and $\int_{C} * d\left(\operatorname{Re} \sum_{n=0}^{\infty} a_{m n}\left(z-\zeta_{m}\right)^{n}\right)=0$. Therefore $\sum_{j=1}^{m} t_{j}=0$ and $h \in H(\hat{\boldsymbol{C}})$. Since $h(\infty)=0$, we conclude that $h \equiv 0$ on $C$, i.e.

$$
u \equiv \sum_{j=1}^{m-1} t_{j}\left(l_{\zeta_{j}}-l_{\zeta_{m}}\right)=\sum_{j=1}^{m-1} t_{j} l_{\zeta_{j}}+\left(-\sum_{j=1}^{m-1} t_{j}\right) l_{\zeta_{m}} .
$$

In view of $\sum_{j=1}^{n} t_{j}=0$, we obtain $u=h_{t}$. Therefore

$$
u(z)=h_{t}(z)=\operatorname{Re}\left(\left(\sum_{j=1}^{m} \zeta_{j} t_{j}\right) z^{-1}+\sum_{n=2}^{\infty} \alpha_{n} z^{-n}\right)
$$

with $\alpha_{n}=n^{-1} \sum_{j=1}^{m} \zeta_{j}^{n} t_{j}$. Since $u \in H_{2}(R)$, (6) implies that $\sum_{j=1}^{m} \zeta_{j} t_{j}=0$. By taking the real and imaginary parts we deduce $A \boldsymbol{t}=\mathbf{0}$, that is $\boldsymbol{t} \in S$. Thus $\boldsymbol{t} \rightarrow h_{t}: S \rightarrow H_{2}(R)$ is a linear bijection and we have established (9). 
3. Suppose $\zeta$ consists of at least four points. Then, since $\operatorname{rank} A(\zeta) \leqq 3$ in any case, we conclude by (9) that $\operatorname{dim} H_{2}(R) \geqq 4-3=1$. Next suppose $\zeta$ consists of three points. Then $\operatorname{dim} H_{2}(R)=3-\operatorname{rank} A(\zeta)$. Although $\operatorname{rank} A(\zeta) \geqq 1$, the equality here cannot occur since otherwise we would have $\zeta_{1}=\zeta_{2}=\zeta_{3}$ in $\zeta$. Therefore rank $A=2$ or 3 . In the latter case, $\operatorname{dim} H_{2}(R)=0$. In the former case, $\operatorname{dim} H_{2}(R)=1$. The relation $\operatorname{rank} A=2$ is, in the present situation, equivalent to $\operatorname{det} A=0$, which in turn is equivalent to $\zeta_{1}, \zeta_{2}$, and $\zeta_{3}$ in $\zeta$ being collinear. If $\zeta$ consists of two points $\zeta_{1}, \zeta_{2}$, then rank $A(\zeta)=2$ since $\zeta_{1} \neq \zeta_{2}$. Therefore $\operatorname{dim} H_{2}(R)=2$-rank $A(\zeta)=0$. If $\zeta$ consists of one point, then $\operatorname{rank} A=1$ and again $\operatorname{dim} H_{2}(R)=0$. The case $\zeta=\emptyset$ (empty) may be treated directly. In this case, in view of (6), any $u \in H_{2}\left(R_{\varnothing}\right)$ must belong to $u \in H(\hat{\boldsymbol{C}})$ and $u(\infty)=0$. Therefore $u \equiv 0$, that is $\operatorname{dim} H_{2}\left(R_{\varnothing}\right)=$ $\operatorname{dim} H_{2}(\boldsymbol{C})=0$.

Note that $R \subset R^{\prime}$ implies $H_{2}\left(R^{\prime}\right) \subset H_{2}(R)$. We have proved:

Theorem. The space $H_{2}(R)$ is degenerate, i.e. $H_{2}(R)=\{0\}$, if and only if $C-R$ consists of at most two points or three noncollinear points.

4. In our earlier paper [3] we considered the degenerate class $O_{H_{2}}$ of Riemannian manifolds $M$ for which $H_{2}(M)=\{0\}$. We also considered the class $O_{S_{2}}$ of those $M$ which contain a subregion $N \in O_{H_{2}}$ with an exterior point. For plane regions $R$ we have thus determined the classes $O_{\mathrm{H}_{2}}$ and $\mathrm{O}_{\mathrm{SH}_{2}}$ as follows: $R \in O_{\mathrm{H}_{2}}$ if and only if $C-R$ contains at most two points or three noncollinear points; $O_{\mathrm{SH}_{2}}=\emptyset$.

5. Consider the set $\zeta=\left\{\zeta_{1}, \zeta_{2}, \zeta_{3}, \ldots, \zeta_{m}\right\}, m \geqq 3$, with the noncollinear points $\zeta_{1}=1+i, \zeta_{2}=1, \zeta_{3}=0$. We have $\operatorname{rank} A=3$ and

$$
\operatorname{dim} H_{2}\left(R_{\zeta}\right)=m-3 .
$$

The set $R^{(k)}=R_{\zeta}$ with $m=3+k$ for $k=0,1,2, \ldots$ satisfies

$$
\operatorname{dim} H_{2}\left(R^{(k)}\right)=k \quad(k=0,1,2, \ldots, \aleph) .
$$

Here $\aleph$ is the countably infinite cardinal number and $R^{(\aleph)}=R_{\zeta}=C-\zeta$ with $\zeta=\left\{1+i, 1,0, \zeta_{4}, \zeta_{5}, \ldots\right\}$ closed and all points in $\zeta$ distinct. We still have to prove (10) for $K=\aleph$. Let $\zeta(m)=\left\{1+i, 1,0, \zeta_{4}, \zeta_{5}, \ldots, \zeta_{m}\right\}(m=4,5, \ldots)$. Observe that area integrals over $R_{\zeta}$ are identical with those over any $R_{\zeta(m)}$ and a fortiori $u \rightarrow u \mid R_{\zeta}$ is an isometric injection: $H_{2}\left(R_{\zeta(m)}\right) \rightarrow H_{2}\left(R_{\zeta}\right)$. Therefore $\operatorname{dim} H_{2}\left(R_{\zeta}\right) \geqq \operatorname{dim} H_{2}\left(R_{\zeta(m)}\right)=$ $m-3$ for every $m=4,5, \ldots$. On the other hand, since $L_{2}(R)$ is separable for any subregion (and actually for any measurable subset) $R$ of $C, \operatorname{dim} H_{2}(R) \leqq \aleph$ as a closed subspace of $L_{2}(R)$ with $\operatorname{dim} L_{2}(R)=\aleph$. We thus deduce (10) also for $k=\aleph$. In sumimary:

Theorem. The dimension of $H_{2}(R)$ for any plane region $R$ is at most countably infinite and there actually exists a plane region $R$ such that the dimension of $H_{2}(R)$ is an arbitrarily preassigned countable cardinal number. 
6. Let $R$ be a plane region and $\zeta \in R$. Then $H_{2}(R)$ is a closed subspace of $H_{2}(R-\zeta)$. We denote by $H_{2}(R)_{\zeta}^{\perp}$ the orthogonal complement of $H_{2}(R)$ in $H_{2}(R-\zeta)$ :

$$
H_{2}(R-\zeta)=H_{2}(R) \oplus H_{2}(R)_{\zeta}^{\perp} .
$$

It may happen that $H_{2}(R)_{\zeta}^{\frac{1}{\zeta}}=\{0\}$. In this case we say that $(R, \zeta)$ is a noneffective pair. Otherwise we assert that $\operatorname{dim} H_{2}(R)_{\zeta}^{\frac{1}{\zeta}}=1$. In fact, suppose $u_{1}, u_{2} \in H_{2}(R)_{\zeta}^{\perp}$. In view of (5)

$$
u_{j}(z)=\operatorname{Re}\left(-c_{j} \log (z-\zeta)+\sum_{n=0}^{\infty} a_{j n}(z-\zeta)^{n}\right)
$$

in a certain $\Delta_{0}(\zeta, \varrho)$, where $c_{j} \in \boldsymbol{R}$ with $c_{j} \neq 0(j=1,2)$. Therefore $c_{2} u_{1}-c_{1} u_{2} \in H_{2}(R)$ and we have $\left(u_{j}, c_{2} u_{1}-c_{1} u_{2}\right)=0(j=1,2)$. From these it follows that $\| c_{2} u_{1}-$ $c_{1} u_{2} \|_{R}^{2}=0$, i.e. $u_{1}$ and $u_{2}$ are linearly dependent. We say that $(R, \zeta)$ is an effective pair if it is not noneffective. For an effective pair $(R, \zeta)$ we have seen that $H_{2}(R) \frac{\downarrow}{\zeta}$ has a single generator:

$$
H_{2}(R)_{\zeta}^{\perp}=R H_{R}(\cdot, \zeta)
$$

where the generator $H_{R}(\cdot, \zeta)$ is so normalized that

$$
H_{R}(z, \zeta)=\operatorname{Re}\left(-\log (z-\zeta)+\sum_{n=0}^{\infty} a_{n}(z-\zeta)^{n}\right) .
$$

7. We next discuss the effectiveness of a point $\zeta$ in a given plane region $R$. There are three cases:

Case 1. $(R, \zeta)$ is a noneffective pair for every $\zeta \in R$.

Case 2. $(R, \zeta)$ is an effective pair for some $\zeta \in R$, noneffective for some other $\zeta \in R$.

Case 3. $(R, \zeta)$ is an effective pair for every $\zeta \in R$.

We call $R$ a weak, unstable, or strong region according as case 1, 2, 3 occurs. We remark that for $R \subset R^{\prime}$, if $R^{\prime}$ is strong so is $R$.

Once more we consider $R_{\zeta}=C-\zeta$ with $\zeta$ the set $\left\{\zeta_{1}, \ldots, \zeta_{m}\right\}$ of distinct points in $\boldsymbol{C}$. Let $\zeta \in R_{\zeta}$ be an arbitrary point and let $\zeta^{\prime}=\zeta \cup\{\zeta\}$. Similarly let $A(\zeta)$ and $A\left(\zeta^{\prime}\right)$ be the matrices (8) associated with $\zeta$ and $\zeta^{\prime}$. Then by (9) we deduce

$$
\operatorname{dim} H_{2}\left(R_{\zeta}\right)_{\zeta}^{\perp}=1-\left(\operatorname{rank} A\left(\zeta^{\prime}\right)-\operatorname{rank} A(\zeta)\right),
$$

and conclude that $\left(R_{\zeta}, \zeta\right)$ is effective if and only if

$$
\operatorname{rank} A(\zeta)=\operatorname{rank} A\left(\zeta^{\prime}\right) \text {. }
$$

Suppose $m \geqq 3$. Recall that $\operatorname{rank} A(\zeta) \geqq 2$. From this we can easily see that (15) is valid for every choice of $\zeta \in R_{\zeta}$ if and only if rank $A(\zeta)=3$, i.e. there are three noncollinear points in $\zeta$. If $m \leqq 1$, then $\zeta^{\prime}$ contains at most two points and therefore $H_{2}\left(R_{\zeta}-\zeta\right)=H_{2}\left(R_{\zeta^{\prime}}\right)=H_{2}\left(R_{\zeta}\right)=\{0\}$ and a fortiori $\operatorname{dim} H_{2}(R)_{\zeta}^{\perp}=0$. In 
the remaining case $\zeta$ contains at least two points and $\zeta$ is collinear. Let $l$ be the line on which every point of $\zeta$ lies. In this case rank $A(\zeta)=2$. If $\zeta \in l-\zeta$, then rank $A\left(\zeta^{\prime}\right)=2$ and $\left(R_{\zeta}, \zeta\right)$ is effective. If $\zeta \notin l$, then $\operatorname{rank} A\left(\zeta^{\prime}\right)=3$ and $\left(R_{\zeta}, \zeta\right)$ is noneffective.

We summarize our observations thus far:

Theorem. A plane region $R$ is strong if and only if $\boldsymbol{C}-\boldsymbol{R}$ contains three noncollinear points, $R$ is weak if and only if $\boldsymbol{C}-\boldsymbol{R}$ contains at most one point, and $R$ is unstable if and only if $\boldsymbol{C}-\boldsymbol{R}$ contains at least two points and is a proper subset of a line.

8. In nos. 8-11 we will concentrate on strong regions $R$. We recall that they are characterized by the existence of three noncollinear points $\zeta_{1}, \zeta_{2}, \zeta_{3}$, in $C-R$. The region $R_{0}=C-\left\{\zeta_{1}, \zeta_{2}, \zeta_{3}\right\}$ is of course strong and by virtue of $R_{0} \supset R$ we have $H_{2}\left(R_{0}\right) \subset H_{2}(R)$. For each $\zeta \in R$ we consider the function

$$
h(z, \zeta)=\left(\sum_{j=1}^{3} t_{j}(\zeta) l_{\zeta_{j}}(z)\right)+l_{\zeta}(z)
$$

where the $t_{j}=t_{j}(\zeta) \in \boldsymbol{R}(j=1,2,3)$ will be later so chosen that $h(\cdot, \zeta) \in H_{2}\left(R_{0}-\zeta\right) \subset$ $H_{2}(R-\zeta)$. Let the Laurent expansion of $h(\cdot, \zeta)$ about $\infty$ be

$$
h(z, \zeta)=\operatorname{Re}\left(-\left(\left(\sum_{j=1}^{3} t_{j}\right)+1\right) \log z+\left(\left(\sum_{j=1}^{3} \zeta_{j} t_{j}\right)+\zeta\right) z^{-1}+\sum_{n=2}^{\infty} \alpha_{n} z^{-n}\right) .
$$

In order that $h(\cdot, \zeta) \in H_{2}\left(R_{0}-\zeta\right)$ it is necessary and sufficient that the $t_{j}=t_{j}(\zeta)$ $(j=1,2,3)$ satisfy the equation

$$
\left(\begin{array}{ccc}
1 & 1 & 1 \\
\operatorname{Re} \zeta_{1} & \operatorname{Re} \zeta_{2} & \operatorname{Re} \zeta_{3} \\
\operatorname{Im} \zeta_{1} & \operatorname{Im} \zeta_{2} & \operatorname{Im} \zeta_{3}
\end{array}\right)\left(\begin{array}{l}
t_{1} \\
t_{2} \\
t_{3}
\end{array}\right)=-\left(\begin{array}{c}
1 \\
\operatorname{Re} \zeta \\
\operatorname{Im} \zeta
\end{array}\right) .
$$

The determinant of the first matrix is nonzero since $\zeta_{1}, \zeta_{2}, \zeta_{3}$ are not collinear. Therefore by the Cramer formula the solution vector with components $t_{j}=t_{j}(\zeta)(j=1,2,3)$ in $\boldsymbol{R}$ satisfying (17) is uniquely determined, and continuous (in fact harmonic) with respect to $\zeta$. The function $h(\cdot, \zeta)$ in (16) with the $t_{j}=t_{j}(\zeta)(j=1,2,3)$ thus determined by (17) belongs to $H_{2}\left(R_{0}-\zeta\right) \subset H_{2}(R-\zeta)$ and we also have $h(z, \cdot) \in H(R-z)$.

9. We next study the continuity of the mapping $\zeta \rightarrow h(z, \zeta): R \rightarrow L_{2}(R)$. We can write

$$
h(z, \zeta)=\operatorname{Re}\left(\sum_{n=2}^{\infty} n^{-1} \alpha_{n}(\zeta) z^{-n}\right)
$$

in some $\Delta_{0}(\infty, \varrho)$ with $\alpha_{n}(\zeta)=\sum_{j=1}^{3} t_{j}(\zeta) \zeta_{j}^{n}+\zeta^{n}(n=2,3, \ldots)$. Suppose $\zeta, \zeta_{j} \in \Delta(0, \sigma)$ : $|z|<\sigma(j=1,2,3)$. Then the continuity of $\zeta \rightarrow t_{j}(\zeta)$ implies the existence of a constant $K_{\sigma}>0$ such that $\left|\alpha_{n}(\zeta)\right| \leqq K_{\sigma} \sigma^{n}(n=2,3, \ldots)$, and we obtain $|h(z, \zeta)| \leqq$ $K_{\sigma} \sum_{n=2}^{\infty} n^{-1} \sigma^{n}|z|^{-n}$ on $\Delta_{0}(\infty, \sigma)$ with $\varrho>\sigma$. Therefore

$$
\|h(\cdot, \zeta)\|_{\Lambda_{0}(\infty, \sigma)} \leqq \sqrt{\pi / 2} K_{\sigma} \sigma^{2} /(\varrho-\sigma) .
$$


Let $\zeta, \zeta^{\prime} \in R$ be contained in $\Delta(0, \sigma), \varrho>\sigma$, and $A=\bar{\Delta}(0, \varrho)-\Delta(0, \sigma)$. Then $\left\|h(\cdot, \zeta)-h\left(\cdot, \zeta^{\prime}\right)\right\|_{R}^{2} \leqq\left\|h(\cdot, \zeta)-h\left(\cdot, \zeta^{\prime}\right)\right\|_{C}^{2}$, which is dominated by the sum of $\left\|h(\cdot, \zeta)-h\left(\cdot, \zeta^{\prime}\right)\right\|_{\Delta(0, \sigma)}^{2},\left\|h(\cdot, \zeta)-h\left(\cdot, \zeta^{\prime}\right)\right\|_{A}^{2}$, and $\left\|h(\cdot, \zeta)-h\left(\cdot, \zeta^{\prime}\right)\right\|_{\Delta_{0}(\infty, e)}^{2}$. The first of these three terms is dominated by

the second by

$$
\left(\sum_{j=1}^{3}\left|t_{j}(\zeta)-t_{j}\left(\zeta^{\prime}\right)\right|\left\|l_{\zeta_{j}}\right\|_{\Delta(0, \sigma)}+\left\|l_{\zeta}-l_{\zeta^{\prime}}\right\|_{\Delta(0, \sigma)}\right)^{2}
$$

$$
\pi\left(\varrho^{2}-\dot{\sigma}^{2}\right)\left(\sup _{z \in A}\left|h(z, \zeta)-h\left(z, \zeta^{\prime}\right)\right|\right)^{2},
$$

and the last by $\left(\|h(\cdot, \zeta)\|_{\Delta_{0}(\infty, \varrho)}+\left\|h\left(\cdot, \zeta^{\prime}\right)\right\|_{\Delta_{0}(\infty, \varrho)}\right)^{2}$, which is not greater than $2 \pi K_{\sigma}^{2} \sigma^{4} /(\varrho-\sigma)^{2}$ by (18). We conclude that

$$
\limsup _{\zeta^{\prime} \rightarrow \zeta}\left\|h(\cdot, \zeta)-h\left(\cdot, \zeta^{\prime}\right)\right\|_{R} \leqq \sqrt{2 \pi} K_{\sigma} \sigma^{2} /(\varrho-\sigma) .
$$

On letting $\varrho \rightarrow+\infty$ we obtain $\lim _{\zeta^{\prime} \rightarrow \zeta}\left\|h(\cdot, \zeta)-h\left(\cdot, \zeta^{\prime}\right)\right\|_{R}=0$.

10. Let $u_{\zeta}(z)=h(z, \zeta)-H_{R}(z, \zeta)$. Clearly $u_{\zeta} \in H_{2}(R)$ and thus the projection of $h(\cdot, \zeta) \in H_{2}(R-\zeta)$ on $H_{2}(R)_{\zeta}^{\frac{1}{}}=\boldsymbol{R} H_{R}(\cdot, \zeta)$ is $H_{R}(\cdot, \zeta)$. Observe that

$$
\left\|h(\cdot, \zeta)-h\left(\cdot, \zeta^{\prime}\right)\right\|_{R}^{2}=\left\|H_{R}(\cdot, \zeta)-H_{R}\left(\cdot, \zeta^{\prime}\right)\right\|_{R}^{2}+\left\|u_{\zeta}-u_{\zeta^{\prime}}\right\|^{2} .
$$

We have obtained the following

Theorem. On a strong region $R$

$$
\lim _{\zeta \rightarrow \zeta^{\prime \prime}}\left\|H_{R}(\cdot, \zeta)-H_{R}\left(\cdot, \zeta^{\prime}\right)\right\|=0
$$

11. Let $R^{\prime}$ and $R^{\prime \prime}$ be subregions of a strong region $R$, hence strong regions. If $R^{\prime} \subset R^{\prime \prime}$, then $H_{R^{\prime \prime}}(\cdot, \zeta)-H_{R^{\prime}}(\cdot, \zeta)$ belongs to $H_{2}\left(R^{\prime}\right)$ and is orthogonal to $H_{R^{\prime}}(\cdot, \zeta)$ over $R^{\prime}$. Therefore

$$
\left\|H_{R^{\prime \prime}}(\cdot, \zeta)-H_{R^{\prime}}(\cdot, \zeta)\right\|_{R^{\prime}}^{2}=\left\|H_{R^{\prime \prime}}(\cdot, \zeta)\right\|_{R^{\prime}}^{2}-\left\|H_{R^{\prime}}(\cdot, \zeta)\right\|_{R^{\prime}}^{2} .
$$

We denote by $\{\Omega\}$ the family of regular subregions $\Omega$ of $R$, a directed set by inclusion, and set $H_{\Omega}(z, \zeta)=0$ for $z \in R-\Omega$. In view of $(20),\left\{\| H_{\Omega}(\cdot, \zeta)_{R}\right\}_{\Omega \in\{\Omega\}}$ is an increasing net and $\left\{H_{\Omega}(\cdot, \zeta)\right\}_{\Omega \in\{\Omega\}}$ is a Cauchy net in $L_{2}(R)$. It is easy to check that the limit is $H_{R}(\cdot, \zeta)$ and

$$
\left\|H_{R}(\cdot, \zeta)-H_{\Omega}(\cdot, \zeta)\right\|_{R}^{2}=\left\|H_{R}(\cdot, \zeta)\right\|_{R}^{2}-\left\|H_{\Omega}(\cdot, \zeta)\right\|_{R}^{2} .
$$

On any compact subset $E$ of $R$, both $\left\|H_{\Omega}(\cdot, \zeta)\right\|_{R}^{2}(\Omega \supset E)$ and $\left\|H_{R}(\cdot, \zeta)\right\|_{R}^{2}$ are continuous (see (19)). Thus the Dini theorem implies:

Theorem. If $R$ is strong, then for any compact subset $E$ of $R$

$$
\lim _{\Omega \rightarrow R}\left(\sup _{\zeta \in E}\left\|H_{R}(\cdot, \zeta)-H_{\Omega}(\cdot, \zeta)\right\|_{R}\right)=0 .
$$


12. We remark that the biharmonic Green's function of the disk $\Delta(0, \varrho)$ is (cf. e.g. Garabedian [2])

$$
\beta_{\Delta(0, \varrho)}(z, \zeta)=\frac{1}{8 \pi}\left[|z-\zeta|^{2} \log \left|\frac{\varrho(z-\zeta)}{\varrho^{2}-\bar{\zeta} z}\right|+\frac{1}{2 \varrho^{2}}\left(|z|^{2}-\varrho^{2}\right)\left(|\zeta|^{2}-\varrho^{2}\right)\right]
$$

on $\Delta(0, \varrho) \times \Delta(0, \varrho)$. Hence, clearly $\boldsymbol{C} \in O_{\beta}$. Similarly, the biharmonic Green's function of the punctured disk $\Delta_{0}(0, \varrho)$ is (cf. [4], [5])

$$
\beta_{\Delta_{0}(0, \varrho)}(z, \zeta)=\beta_{\Delta(0, \varrho)}(z, \zeta)-\left|6 \pi \varrho^{-2} \beta_{\Delta(0, \varrho)}(z, 0) \beta_{\Delta(0, \varrho)}(\zeta, 0)\right|
$$

on $\Delta_{0}(0, \varrho) \times \Delta_{0}(0, \varrho)$. Therefore $C-\{0\} \in O_{\beta}$.

In view of the above, relation (4), and the theorem in no. 7, the assertions $1^{\circ}$ and $3^{\circ}$ can easily be deduced. By nos. 9 and $\mathbf{1 0}$ the first three assertions in $2^{\circ}$ are clear. Since $\beta_{\Omega}(\cdot, \zeta)$ is biharmonic on $\Omega-\zeta$ and uniformly convergent to $\beta_{R}(\cdot, \zeta)$ on every compact subset of $R-\zeta, \beta_{R}(\cdot, \zeta)$ is also biharmonic on $R-\zeta$.

\section{References}

[1] Bergman, S., and M. Schiffer: Kernel functions and elliptic differential equations in mathe matical physics. - Pure and Applied Mathematics IV, Academic Press Inc., Publishers New York, N. Y., 1953.

[2] Garabedian, P. R.: Partial differential equations. - John Wiley \& Sons, Inc., New YorkLondon-Sydney, 1964.

[3] Nakai, M., and L. Sario: A nonexistence test for biharmonic Green's functions of clamped bodies. - Math. Scand. 40, 1977, 151-160.

[4] NAKaI, M., and L. SARIO: Green's functions of the clamped punctured disk. - J. Austral. Math. Soc. Ser. B 20, 1977 (to appear).

[5] Sario, L., M. Nakai, C. Wang, and L. Chung: Classification theory of Riemannian manifolds. Harmonic, quasiharmonic, and biharmonic functions. - Lecture Notes in Mathematics 605, Springer-Verlag, Berlin-Heidelberg-New York, 1977.

Nagoya Institute of Technology

Department of Mathematics

Gokiso, Shôwa

Nagoya 466

Japan
University of California, Los Angeles

Department of Mathematics

Los Angeles, California 90024

USA

Received 16 May 1978 